\title{
Métodos de establecimiento de pasturas en zonas áridas de México utilizando semillas crudas o cariópsides Methods for pasture establishment in arid zones of Mexico using crude seeds or caryopses
}

\author{
ADRIÁN RAYMUNDO QUERO-CARRILLO ${ }^{1}$, FILOGONIO JESÚS HERNÁNDEZ-GUZMÁN², MAURICIO \\ VELÁZQUEZ-MARTÍNEZ ${ }^{3}$, HÉCTOR GUILLERMO GÁMEZ-VÁZQUEZ ${ }^{3}$, PATRICIA LANDA-SALGADO ${ }^{4}$ Y \\ PEDRO AGUILAR-LÓPEZ ${ }^{2}$ \\ ${ }^{1}$ Ganadería, Colegio de Postgraduados, Texcoco de Mora, Estado de México, México. www.colpos.mx \\ ${ }^{2}$ Universidad Politécnica Francisco I. Madero, Tepatepec, Hidalgo, México. www.upfim.edu.mx \\ ${ }^{3}$ Campo Experimental San Luis, Instituto Nacional de Investigaciones Forestales, Agrícolas y Pecuarias (INIFAP), \\ San Luis Potosí, México. www.inifap.gob.mx \\ ${ }^{4}$ Universidad Autónoma Chapingo, Texcoco de Mora, Estado de México, México. www.chapingo.mx
}

Palabras clave: Densidad de siembra, gramíneas introducidas, gramíneas nativas, suelo apisonado, tapado de semilla. Keywords: Harrowing, introduced grasses, native grasses, plant density, seedbed rolling.

\section{Resumen}

En 2 sitios del Desierto Chihuahuense, México, se evaluó el establecimiento en secano de las gramíneas nativas Banderita (Bouteloua curtipendula) y Navajita (B. gracilis) y las introducidas Buffel (Cenchrus ciliaris) y Rhodes (Chloris gayana), utilizando cariópsides y/o semillas crudas (semilla limpia con brácteas y aristas) y 4 métodos de tapado. Los sitios de siembra fueron Atotonilco El Grande, Hidalgo y Salinas Hidalgo, San Luis Potosí. Las siembras se hicieron a voleo a razón de 1,000 cariópsides y/o semillas crudas viables $/ \mathrm{m}^{2}$. Los métodos de tapado fueron: paso de rastra con ramas; rodillo; rastra con ramas + rodillo; y sin tapado (testigo). Las variables de respuesta incluyeron número de plantas emergidas y de plantas establecidas, diámetro de corona, altura de planta y número de tallos por planta. Se utilizó un diseño completamente al azar con arreglo factorial 2 × 2 × 4 con 3 repeticiones. No se observaron diferencias entre sitios y se establecieron, en promedio, 2 plantas $/ \mathrm{m}^{2}$. Con las especies nativas (Banderita y Navajita) se obtuvo mayor cantidad de plantas emergidas y establecidas cuando la siembra se hizo con semillas crudas, mientras que en introducidas no se encontró diferencia entre siembra con semilla cruda y siembra con cariópsides. Cuando se utilizó el método de tapado y apisonado del suelo se observaron mayor diámetro de corona y altura de planta. El mayor número de plantas establecidas se obtuvo en pasturas de Navajita y Rhodes. En las especies nativas la eliminación de brácteas accesorias en las semillas no se tradujo en mejor establecimiento, mientras que en las gramíneas introducidas esta práctica sí mejoró el establecimiento. En ambos grupos de especies el apisonado mejoró el establecimiento.

\begin{abstract}
Correspondence: F.J. Hernández-Guzmán, Universidad Tecnológica de Tulancingo, Camino a Ahuehuetitla \# 301 Col. Las Presas, Tulancingo 43642, Hidalgo, México.

Email: fjesushg@ hotmail.com
\end{abstract}

In order to evaluate the establishment of the native grasses Sideoats grama ('Banderita', Bouteloua curtipendula) and Blue grama ('Navajita', B. gracilis) and the introduced grasses buffel (Cenchrus ciliaris) and Rhodes (Chloris gayana) at 2 sites in the Chihuahuan Desert, the use of caryopses vs. entire seeds (diaspores) was studied applying 4 methods of covering after broadcasting. The sites were Atotonilco El Grande, Hidalgo State; and Salinas Hidalgo, San Luis Potosí State, Mexico. One thousand viable seeds (caryopses or diaspores) were sown per square meter. 
The 4 methods of covering were: harrowing with a tree branch; use of a roller; use of a tree branch harrow plus roller; and no covering (control). The response variables measured were number of emerged seedlings; number of established plants; diameter of the crown; plant height; and number of stems per plant. The design was a completely randomized $2 \times 2 \times 4$ factorial arrangement with 3 replicates at each site. There were no differences between sites and, on average, 2 plants $/ \mathrm{m}^{2}$ were established. With the native species greater numbers of emerged and established plants were obtained by using diaspores while with introduced species there were no significant differences between caryopses and diaspores. Harrowing with a tree branch and/or rolling the soil resulted in thicker crowns and greater plant height. The highest number of stems per established plant were recorded in Blue grama and Rhodes grass. In native grasses it is important not to remove accessory bracts, in contrast with introduced grasses. In both species groups establishment is improved by rolling.

\section{Introducción}

El éxito de establecimiento por semilla de pasturas en secano depende, entre otros factores, del porcentaje y vigor de germinación de ésta, la preparación del suelo y profundidad de siembra, y de las condiciones de clima. Por tanto, para la rehabilitación exitosa de pasturas es necesario que la industria de semillas ofrezca al productor máxima calidad genética, física y biológica de éstas, aspectos influenciados por el ambiente de producción, cosecha, beneficio y almacenamiento de la semilla (Probert y Hay 2000). Es necesario que la semilla enfrente solamente los factores modificadores (entre los cuales los siguientes son particularmente frecuentes en zonas áridas: sequías intraestivales mayores a 4 semanas; lluvias torrenciales que arrastran la semilla; y formación de costra que impide la emergencia), ante un buen manejo de la siembra de praderas de secano con semilla de calidad destacada (Quero-Carrillo et al. 2014).

En zonas áridas y bajo condiciones de humedad adecuadas, los cambios de temperatura son el principal limitante para establecer pasturas de temporal. Se ha encontrado que fluctuaciones de temperaturas nocturnas $\left(10-20{ }^{\circ} \mathrm{C}\right)$ y diurnas $\left(20-30{ }^{\circ} \mathrm{C}\right)$ afectan marcadamente el porcentaje de germinación (Evans y Young 1987; Esqueda et al. 2002; Larsen y Bibby 2004). Según Ward et al. (2006) las cariópsides del pasto Buffel (Cenchrus ciliaris) requieren $12.5{ }^{\circ} \mathrm{C}$ y $15-20 \mathrm{~mm}$ de precipitación para alcanzar $60 \%$ de germinación. Por su parte Tian et al. (2002) encontraron bajos porcentajes de germinación en Tripsacum dactyloides cuando utilizaron semillas completas con brácteas de la espiguilla (glumas, lemas, paleas, ramillas y aristas). No obstante cuando estas brácteas fueron eliminadas la germinación fue $10 \%$ mayor y alcanzó un valor de $90 \%$ cuando se utilizaron cariópsides escarificadas.

Otro factor importante para garantizar un buen establecimiento de pasturas empleando semillas es la preparación de la cama de siembra. Cox et al. (1986) encontraron un mejor establecimiento y producción de forraje en pasturas de zonas áridas con dos pases de rastra y siembra mecanizada.

En el Desierto Chihuahuense, México, los pastizales nativos cubren aproximadamente $15 \%$ de la superficie (PMAR 2012). Estos pastizales nativos son especialmente vulnerables frente al cambio climático (Townsend et al. 2002) y su rehabilitación con especies perennes es una alternativa a mediano plazo. Sin embargo, la comparación entre los métodos de siembra más utilizados y entre los materiales de siembra utilizados [cariópsides o semillas crudas (semilla limpia con aristas)] no está documentada.

El objetivo de este estudio fue evaluar la emergencia y el establecimiento de especies de gramíneas nativas o introducidas utilizando semillas crudas o solo cariópsides en 4 métodos de tapado en 2 sitios del Desierto Chihuahuense, México.

\section{Materiales y Métodos}

El estudio se realizó entre el 25 de junio de 2010 y el 25 de octubre de 2011 en los municipios de Atotonilco El Grande, Estado de Hidalgo (20²4' N, 98 $43^{\prime}$ O) y Salinas Hidalgo, Estado de San Luis Potosí (22 $43^{\prime}$ N, $101^{\circ} 35^{\prime}$ O), México. Atotonilco El Grande se encuentra a 1,965 msnm en un clima templado-seco [BSk según la clasificación de Köppen (Kottek et al. 2006)] caracterizado por una temperatura media anual de $16{ }^{\circ} \mathrm{C}$ y 560 $\mathrm{mm}$ de precipitación anual; el suelo es un Vertisol (INEGI 1984). Salinas Hidalgo se encuentra a 2,100 msnm, igualmente en clima templado-seco (BSk) con una temperatura media anual de $16.2{ }^{\circ} \mathrm{C}$ y precipitación anual de $336 \mathrm{~mm}$ (INEGI 2012), con predominio de suelo Castañozem cálcico (FAO-UNESCO-ISRIC 1988) de textura arcillosa. Se evaluaron 2 especies de gramíneas nativas del Desierto Chihuahuense: Banderita [Bouteloua curtipendula (Michx.) Torr.] y 
Navajita [Bouteloua gracilis (Kunth) Lag. ex Griffiths] y 2 especies introducidas de África: los pastos Buffel [Cenchrus ciliaris L.; ahora: Pennisetum ciliare (L.) Link], variedad Común (o T4464 o americano) y Rhodes (Chloris gayana Kunth), variedad Bell.

La preparación de los suelos en ambos sitios consistió en voltearlo con arado tipo barbecho y después 2 pasos de rastra. Las cariópsides se obtuvieron a partir de semillas comerciales adquiridas en enero 2009 las cuales fueron friccionadas en forma manual hasta obtener aproximadamente $1 \mathrm{~kg}$ de cada especie. Las cariópsides pequeñas y rotas fueron eliminadas con paso por tamiz de $0.42 \mathrm{~mm}$ y mediante microscopio estereoscópico se eliminaron cariópsides dañadas. Las cariópsides resultantes fueron analizadas en laboratorio con el fin de determinar las condiciones fisiológicas y físicas de acuerdo con las normas ISTA (1996); un protocolo similar se siguió con las semillas crudas, constituidas por cariópsides más glumas, lemas, paleas, ramillas modificadas y aristas. Para la siembra se utilizaron 1,000 semillas botánicas germinables por $\mathrm{m}^{2}$ de cada una de ellas (Cuadro 1).

En cada sitio experimental se establecieron 96 parcelas de $35 \mathrm{~m}^{2}$ cada una (7 x $5 \mathrm{~m}$ ): 48 para las especies nativas y 48 para las introducidas. En Atotonilco El Grande la siembra se hizo el 25 de junio de 2010 y en Salinas Hidalgo el 5 de julio de 2010, en suelos a capacidad de campo. Las siembras se hicieron a voleo y se utilizaron 4 métodos de tapado: (1) un paso de rastra acondicionada con ramas; (2) apisonado con rodillo (compactación); (3) rastra con ramas + apisonado; y (4) semilla sin cubrir (testigo). El objetivo del apisonado fue favorecer un mayor contacto del suelo con las semillas, para lo cual se usó como rodillo un cilindro con peso de $80 \mathrm{~kg}$.
En ambos sitios la evaluación de las plantas emergidas se hizo entre el 20 y 24 de octubre de 2010 y de las plantas establecidas entre el 24 y 28 de octubre de 2011. No se aplicaron fertilizantes, insecticidas o herbicidas ni se defoliaron las plantas durante el experimento. El análisis de los datos se hizo teniendo en cuenta el origen de las especies (nativas vs. introducidas). En cada sitio se utilizó un Diseño Completamente al Azar con arreglo factorial $2 \times 2 \times 4$ con 3 repeticiones. Los tratamientos incluyeron 2 especies, nativas o introducidas, ambas con la combinación de 2 tipos de material de siembra (semillas crudas y cariópsides) y 4 métodos de siembra, en 2 sitios, es decir, 16 tratamientos por especies nativas e igual número por especies introducidas.

Las variables de respuesta analizadas en cada unidad experimental (parcela) incluyeron número de plantas emergidas y establecidas, con base en 3 muestreos al azar en cada parcela, en rectángulos de $30 \times 60 \mathrm{~cm}$. Por otro lado, en 15 plantas tomadas al azar en cada unidad experimental se midió: diámetro de corona $(\mathrm{mm})$; altura de planta $(\mathrm{cm}$, desde el nivel del suelo hasta el ápice de la inflorescencia o de la hoja bandera); y número de tallos por planta.

En el Cuadro 2 aparecen los registros de temperatura, precipitación y radiación global de estaciones meteorológicas del INIFAP, localizadas a $37 \mathrm{~km}$ del sitio experimental en Atotonilco El Grande y a $30 \mathrm{~km}$ del sitio en Salinas Hidalgo.

Los datos de todas las variables se transformaron mediante $\sqrt{x+0.5}$ y después se analizaron con el programa GLM de SAS (2009) y prueba de Tukey.

Cuadro 1. Caracterización física y fisiológica de las unidades de dispersión (semillas crudas y cariópsides) de 4 especies de gramíneas.

\begin{tabular}{|c|c|c|c|c|c|c|c|c|c|c|c|}
\hline \multirow[t]{2}{*}{ Especie } & \multicolumn{8}{|c|}{ Características físicas } & \multicolumn{3}{|c|}{ Características fisiológicas } \\
\hline & 1 & 2 & 3 & 4 & 5 & 6 & 7 & 8 & 9 & 10 & 11 \\
\hline Navajita (Bouteloua gracilis) & 86 & 62 & 1,500 & 1.980 & 1.6 & 0.6 & 1,037 & 1,094 & 84 & 78 & 82 \\
\hline Banderita (B. curtipendula) & 95 & 23 & 992 & 1,532 & 5 & 0.75 & 1,040 & 1,048 & 97 & 92 & 94 \\
\hline Buffel (Cenchrus ciliaris) & 61 & 40 & 386 & 2,015 & 60 & 2.3 & 1,074 & 1,079 & 86 & 19 & 24 \\
\hline Rhodes (Chloris gayana) & 82 & 32 & 2,417 & 3,858 & 2.6 & 0.35 & 1,049 & 1,072 & 88 & 63 & 81 \\
\hline
\end{tabular}

1: Pureza $(\%)=$ Porcentaje de semilla de la especie en cuestión; 2: Número de cariópsides en 100 semillas crudas; 3: Número de semillas crudas en un gramo; 4: Número de cariópsides en un gramo; 5: Dosis de siembra de semillas crudas (g/m²); 6: Dosis de siembra cariópsides $\left(\mathrm{g} / \mathrm{m}^{2}\right) ; 7$ : Número de plantas esperadas $/ \mathrm{m}^{2}$, sembradas con semillas crudas; 8: Número de plantas esperadas $/ \mathrm{m}^{2}$, sembradas con cariópsides con base en semilla pura germinable; 9: Viabilidad (\%; tetrazolio 0.1\%); 10: Germinación de semillas crudas (\%); 11: Germinación de cariópsides (\%). 
Cuadro 2. Temperatura, precipitación anual y radiación global en 2 municipios del Desierto Chihuahuense, México.

\begin{tabular}{|c|c|c|c|c|c|c|c|c|c|}
\hline \multirow[t]{2}{*}{ Año } & \multirow[t]{2}{*}{ Época } & \multicolumn{4}{|c|}{ Salinas Hidalgo (San Luis Potosí) } & \multicolumn{4}{|c|}{ Atotonilco El Grande (Hidalgo) } \\
\hline & & $\begin{array}{c}\text { Temp. } \\
\text { mín. }\left({ }^{\circ} \mathrm{C}\right)\end{array}$ & $\begin{array}{c}\text { Temp. } \\
\text { media }\left({ }^{\circ} \mathrm{C}\right)\end{array}$ & $\begin{array}{l}\text { Prec. } \\
(\mathrm{mm})\end{array}$ & $\begin{array}{c}\text { Radiación } \\
\left(\mathrm{W} / \mathrm{m}^{2}\right)\end{array}$ & $\begin{array}{c}\text { Temp. } \\
\text { mín. }\left({ }^{\circ} \mathrm{C}\right)\end{array}$ & $\begin{array}{c}\text { Temp. } \\
\text { media }\left({ }^{\circ} \mathrm{C}\right)\end{array}$ & $\begin{array}{l}\text { Prec. } \\
(\mathrm{mm})\end{array}$ & $\begin{array}{c}\text { Radiación } \\
\left(\mathrm{W} / \mathrm{m}^{2}\right)\end{array}$ \\
\hline \multirow[t]{4}{*}{2010} & Primavera & $1^{1}$ & $10^{1}$ & $381^{2}$ & $531^{2}$ & $7.2^{1}$ & $13^{1}$ & $81^{2}$ & $471^{2}$ \\
\hline & Verano & 9 & 19 & 4 & 596 & 13 & 19 & 35 & 509 \\
\hline & Otoño & 14 & 19 & 22 & 546 & 13 & 17 & 120 & 390 \\
\hline & Invierno & 0 & 12 & 0 & 489 & 7 & 13 & 13 & 511 \\
\hline \multirow[t]{4}{*}{2011} & Primavera & 3 & 15 & 0 & 481 & 8 & 15 & 1 & 556 \\
\hline & Verano & 9 & 21 & 4 & 581 & 13 & 19 & 28 & 510 \\
\hline & Otoño & 12 & 19 & 41 & 532 & 12 & 16 & 114 & 467 \\
\hline & Invierno & 2 & 13 & 5 & 519 & 8 & 14 & 16 & 477 \\
\hline \multicolumn{2}{|c|}{ Total/Promedio } & 6 & 16 & 457 & 4,275 & 10 & 16 & 408 & 3,891 \\
\hline
\end{tabular}

${ }^{1}$ Promedio de datos en la época del año. ${ }^{2}$ Suma de datos en la época del año. Prec. = Precipitación.

\section{Resultados}

Número de plantas emergidas y establecidas en especies nativas

En Atotonilco El Grande se observaron diferencias $(\mathrm{P}<0.01)$ tanto para plantas emergidas como para establecidas. Se encontró efecto significativo tanto del material de siembra (semilla cruda o cariópsides) como del método de tapado ( $\mathrm{P}<0.001$; Cuadro 3 ) como lo demuestra el hecho que el mayor número de plantas se registró cuando la siembra se hizo con semillas crudas y pase de rodillo en comparación con los demás tratamientos. El número de plantas establecidas fue afectado por la especie $(\mathrm{P}<0.05)$ y por el material de siembra $(\mathrm{P}<0.01)$ : El pasto Navajita presentó un mayor número de plantas establecidas que Banderita y se observó un mayor número de plantas en siembras con semillas crudas que con cariópsides.

En Salinas Hidalgo se observaron diferencias entre tratamientos tanto para plantas emergidas como para establecidas $(\mathrm{P}<0.01)$. El pasto Banderita presentó mayor número de plantas emergidas que Navajita $(\mathrm{P}<0.01)$ (Cuadro 3). Por otra parte, el número de plantas establecidas fue mayor cuando se utilizó semilla cruda que cuando se utilizó cariópsides $(\mathrm{P}<0.05)$. El mayor número de plantas emergidas se observó cuando la semilla fue cubierta con ramas $(\mathrm{P}<0.01)$. El número de plantas establecidas en Banderita fue más alto que en Navajita $(\mathrm{P}<0.01)$; igualmente fue más alto cuando se utilizó semilla cruda que cuando se utilizó cariópsides $(\mathrm{P}<0.001)$ o cuando se utilizó ramas más rodillo o solo rodillo vs. el tratamiento testigo.

Cuadro 3. Plantas emergidas y establecidas (número por metro cuadrado) de 2 gramíneas nativas en 2 localidades del Desierto Chihuahuense, México, en diferentes sistemas de siembra.

\begin{tabular}{|c|c|c|c|c|}
\hline \multirow[t]{2}{*}{ Tratamiento } & \multicolumn{2}{|c|}{ Atotonilco El Grande, Hidalgo } & \multicolumn{2}{|c|}{ Salinas Hidalgo, San Luis Potosí } \\
\hline & Plantas emergidas & Plantas establecidas & Plantas emergidas & Plantas establecidas \\
\hline \multicolumn{5}{|l|}{ Especie } \\
\hline Navajita & $10 a^{1}$ & $2.3 \mathrm{a}$ & $3.4 \mathrm{~b}$ & $0.6 b$ \\
\hline Banderita & $10 \mathrm{a}$ & $1.8 \mathrm{~b}$ & $5.3 \mathrm{a}$ & $1.5 \mathrm{a}$ \\
\hline \multicolumn{5}{|l|}{ Material de siembra } \\
\hline Semillas crudas & $17 \mathrm{a}$ & $2.5 \mathrm{a}$ & $7.2 \mathrm{a}$ & $1.7 \mathrm{a}$ \\
\hline Cariópsides & $3 b$ & $1.6 \mathrm{~b}$ & $1.6 \mathrm{~b}$ & $0.4 \mathrm{~b}$ \\
\hline \multicolumn{5}{|l|}{ Método de tapado } \\
\hline Testigo & $8 b$ & $1.7 \mathrm{a}$ & $1.9 \mathrm{c}$ & $0.1 \mathrm{~b}$ \\
\hline Ramas & $10 \mathrm{~b}$ & $2.4 \mathrm{a}$ & $5.8 \mathrm{a}$ & $1.8 \mathrm{a}$ \\
\hline Rodillo & $11 \mathrm{a}$ & $1.8 \mathrm{a}$ & $4.8 \mathrm{bc}$ & $0.9 \mathrm{a}$ \\
\hline Ramas + rodillo & $10 \mathrm{~b}$ & $2.3 \mathrm{a}$ & $4.9 \mathrm{ab}$ & $1.4 \mathrm{a}$ \\
\hline
\end{tabular}

${ }^{1}$ Valores en una misma columna y tratamiento seguidos de letras iguales no difieren en forma significativa $(\mathrm{P}<0.05)$. 
Cuadro 4. Plantas emergidas y establecidas (número por metro cuadrado) de 2 gramíneas introducidas en 2 localidades del Desierto Chihuahuense, México, en diferentes sistemas de siembra.

\begin{tabular}{|c|c|c|c|c|}
\hline \multirow[t]{2}{*}{ Tratamiento } & \multicolumn{2}{|c|}{ Atotonilco El Grande, Hidalgo } & \multicolumn{2}{|c|}{ Salinas Hidalgo, San Luis Potosí } \\
\hline & Plantas emergidas & Plantas establecidas & Plantas emergidas & Plantas establecidas \\
\hline \multicolumn{5}{|l|}{ Especie } \\
\hline Buffel & $5 b^{1}$ & $2 \mathrm{a}$ & $11 \mathrm{a}$ & $2 \mathrm{a}$ \\
\hline Rhodes & $17 \mathrm{a}$ & $3 a$ & $7 \mathrm{~b}$ & $2 \mathrm{a}$ \\
\hline \multicolumn{5}{|l|}{ Material de siembra } \\
\hline Semilla cruda & $10 \mathrm{a}$ & $3 a$ & $15 \mathrm{a}$ & $3 a$ \\
\hline Cariópsides & $11 \mathrm{a}$ & $2 b$ & $3 b$ & $0.5 b$ \\
\hline \multicolumn{5}{|l|}{ Método de tapado } \\
\hline Testigo & $10 \mathrm{a}$ & $2 \mathrm{a}$ & $10 \mathrm{a}$ & $1 \mathrm{a}$ \\
\hline Ramas & $11 \mathrm{a}$ & $3 a$ & $8 \mathrm{a}$ & $2 \mathrm{a}$ \\
\hline Rodillo & $11 \mathrm{a}$ & $2 \mathrm{a}$ & $11 \mathrm{a}$ & $2 \mathrm{a}$ \\
\hline Ramas + rodillo & $11 \mathrm{a}$ & $3 a$ & $8 \mathrm{a}$ & $2 \mathrm{a}$ \\
\hline
\end{tabular}

${ }^{1}$ Valores en una misma columna y tratamiento seguidos de letras iguales no difieren en forma significativa $(\mathrm{P}<0.05)$.

\section{Número de plantas emergidas y establecidas en especies} introducidas

En Atotonilco El Grande se observaron diferencias entre los tratamientos para ambas variables $(\mathrm{P}<0.001)$ (Cuadro 4): El número de plantas emergidas fue mayor en Rhodes que en Buffel y el número de plantas establecidas fue mayor cuando la siembra se hizo por semilla cruda.

También en Salinas Hidalgo se registraron diferencias entre los tratamientos para ambas variables $(\mathrm{P}<0.001)$. En plantas emergidas se observó efecto de especie $(\mathrm{P}<0.01)$ y de material de siembra $(\mathrm{P}<0.001)$ (Cuadro 4) siendo mayores los valores en Buffel y siembra por semilla cruda en comparación con Rhodes y siembra por cariópsides. En plantas establecidas se observó solo un efecto de material de siembra: El número de plantas fue mayor cuando se utilizó semilla cruda $(\mathrm{P}<0.001)$.
Diámetro de corona, altura de planta y número de tallos en especies nativas

En Atotonilco El Grande se observaron diferencias para estas características tanto en plantas emergidas como establecidas $(\mathrm{P}<0.001)$. Banderita presentó los valores más altos de diámetro de corona y altura de planta (Cuadro 5), mientras que Navajita presentó el mayor número de tallos $(\mathrm{P}<0.001)$. Tanto las plantas emergidas como las establecidas presentaron mayor diámetro de corona cuando la siembra se hizo con cariópsides $(\mathrm{P}<0.01)$. Igualmente el diámetro de corona de plantas emergidas fue más alto cuando la semilla fue tapada o se pasó rodillo después de la siembra $(\mathrm{P}<0.01)$. El apisonado también aumentó el diámetro de corona en plantas establecidas $(\mathrm{P}<0.05)$.

Cuadro 5. Diámetro de corona, altura de planta y número de tallos por planta de 2 gramíneas nativas del Desierto Chihuahuense, en Atotonilco el Grande, Hidalgo, México.

\begin{tabular}{|c|c|c|c|c|c|c|}
\hline \multirow[t]{2}{*}{ Tratamiento } & \multicolumn{3}{|c|}{ Plantas emergidas } & \multicolumn{3}{|c|}{ Plantas establecidas } \\
\hline & $\begin{array}{l}\text { Diám. corona } \\
(\mathrm{mm})\end{array}$ & $\begin{array}{l}\text { Altura planta } \\
(\mathrm{cm})\end{array}$ & $\begin{array}{l}\text { Tallos por } \\
\text { planta (no.) }\end{array}$ & $\begin{array}{l}\text { Diám. corona } \\
(\mathrm{mm})\end{array}$ & $\begin{array}{l}\text { Altura planta } \\
(\mathrm{cm})\end{array}$ & $\begin{array}{c}\text { Tallos por } \\
\text { planta (no.) }\end{array}$ \\
\hline \multicolumn{7}{|l|}{ Especie } \\
\hline Navajita & $2.8 \mathrm{~b}^{1}$ & $3.4 \mathrm{~b}$ & $11 \mathrm{a}$ & $3.8 \mathrm{~b}$ & $14.8 \mathrm{~b}$ & $75 \mathrm{a}$ \\
\hline Banderita & $4.0 \mathrm{a}$ & $11.1 \mathrm{a}$ & $6 b$ & $6.4 \mathrm{a}$ & $31.3 \mathrm{a}$ & $33 b$ \\
\hline \multicolumn{7}{|l|}{ Material de siembra } \\
\hline Semilla cruda & $3.2 \mathrm{~b}$ & $7.9 \mathrm{a}$ & $9 \mathrm{a}$ & $4.7 \mathrm{~b}$ & $29.7 \mathrm{a}$ & $46 a$ \\
\hline Cariópsides & $3.6 \mathrm{a}$ & $7.6 \mathrm{a}$ & $8 \mathrm{a}$ & $5.5 \mathrm{a}$ & $23.3 \mathrm{a}$ & $52 \mathrm{a}$ \\
\hline \multicolumn{7}{|l|}{ Método de tapado } \\
\hline Testigo & $2.9 \mathrm{~b}$ & $6.8 \mathrm{a}$ & $9 \mathrm{a}$ & $3.8 \mathrm{~b}$ & $23.3 \mathrm{a}$ & $56 a$ \\
\hline Ramas & $3.5 \mathrm{ab}$ & $7.2 \mathrm{a}$ & $9 \mathrm{a}$ & $4.8 \mathrm{~b}$ & $24.8 \mathrm{a}$ & $59 \mathrm{a}$ \\
\hline Rodillo & $3.5 \mathrm{ab}$ & $9.4 \mathrm{a}$ & $8 \mathrm{a}$ & $5.8 \mathrm{a}$ & $36.1 \mathrm{a}$ & $31 \mathrm{a}$ \\
\hline Ramas + rodillo & $3.8 \mathrm{a}$ & 7.6a & $9 \mathrm{a}$ & $6.0 \mathrm{a}$ & $21.8 \mathrm{a}$ & $51 \mathrm{a}$ \\
\hline
\end{tabular}

${ }^{1}$ Valores en una misma columna y tratamiento seguidos de letras iguales no difieren en forma significativa $(\mathrm{P}<0.05)$. 
Cuadro 6. Diámetro de corona, altura de planta y número de tallos por planta de 2 gramíneas nativas del Desierto Chihuahuense, en Salinas Hidalgo, San Luis Potosí, México.

\begin{tabular}{|c|c|c|c|c|c|c|}
\hline \multirow[t]{2}{*}{ Tratamiento } & \multicolumn{3}{|c|}{ Plantas emergidas } & \multicolumn{3}{|c|}{ Plantas establecidas } \\
\hline & $\begin{array}{c}\text { Diám. corona } \\
(\mathrm{mm})\end{array}$ & $\begin{array}{l}\text { Altura planta } \\
(\mathrm{cm})\end{array}$ & $\begin{array}{l}\text { Tallos por } \\
\text { planta (no.) }\end{array}$ & $\begin{array}{l}\text { Diám. corona } \\
(\mathrm{mm})\end{array}$ & $\begin{array}{c}\text { Altura planta } \\
(\mathrm{cm})\end{array}$ & $\begin{array}{l}\text { Tallos por } \\
\text { planta (no.) }\end{array}$ \\
\hline \multicolumn{7}{|l|}{ Especie } \\
\hline Navajita & $3.0 b^{1}$ & $4.2 \mathrm{~b}$ & $13 \mathrm{a}$ & $10.7 \mathrm{~b}$ & $12.3 b$ & $79 a$ \\
\hline Banderita & $3.8 \mathrm{a}$ & $12.8 \mathrm{a}$ & $9 b$ & $17.9 \mathrm{a}$ & $28.0 \mathrm{a}$ & $32 b$ \\
\hline \multicolumn{7}{|l|}{ Material de siembra } \\
\hline Semilla cruda & $3.5 \mathrm{a}$ & 9.9a & $11 \mathrm{a}$ & $13.6 \mathrm{~b}$ & $29.5 \mathrm{a}$ & $57 \mathrm{a}$ \\
\hline Cariópsides & $3.4 \mathrm{a}$ & $8.5 \mathrm{a}$ & $12 \mathrm{a}$ & $14.9 \mathrm{a}$ & $20.1 \mathrm{~b}$ & $54 \mathrm{a}$ \\
\hline \multicolumn{7}{|l|}{ Método de tapado } \\
\hline Testigo & $3.2 \mathrm{a}$ & $8.1 \mathrm{a}$ & $9 b$ & $12.6 \mathrm{c}$ & $20.2 \mathrm{a}$ & $51 \mathrm{a}$ \\
\hline Ramas & $3.4 \mathrm{a}$ & $8.2 \mathrm{a}$ & $11 \mathrm{a}$ & $13.9 \mathrm{bc}$ & $19.2 \mathrm{a}$ & $57 \mathrm{a}$ \\
\hline Rodillo & $3.3 \mathrm{a}$ & $11.5 \mathrm{a}$ & $13 \mathrm{a}$ & $14.7 \mathrm{ab}$ & $38.8 \mathrm{a}$ & $57 \mathrm{a}$ \\
\hline Ramas + rodillo & $3.8 \mathrm{a}$ & $9.1 \mathrm{a}$ & $13 a$ & $16.0 \mathrm{a}$ & $21.2 \mathrm{a}$ & $57 \mathrm{a}$ \\
\hline
\end{tabular}

${ }^{1}$ Valores en una misma columna y tratamiento seguidos de letras iguales no difieren en forma significativa $(\mathrm{P}<0.05)$.

En Salinas Hidalgo se observaron diferencias en diámetro de corona, altura de planta y número de tallos tanto en las plantas emergidas como en las establecidas $(\mathrm{P}<0.01)$. En las primeras, el pasto Banderita presentó los mayores valores de estas características (Cuadro 6). En plantas establecidas de Banderita se observó un mayor valor de diámetro de corona y altura de planta, pero menor número de tallos que en Navajita. En plantas establecidas Banderita presentó un mayor diámetro de corona y altura de planta, pero menor número de tallos que Navajita $(\mathrm{P}<0.001)$. La siembra por cariópsides resultó en mayor diámetro de corona $(\mathrm{P}<0.05)$ pero en menor altura de planta $(\mathrm{P}<0.001)$. Tapar la semilla después de la siembra y apisonarla favoreció un mayor diámetro de corona $(\mathrm{P}<0.05)$.
Diámetro de corona, altura de planta y número de tallos en especies introducidas

En Atotonilco El Grande se observaron diferencias para estas características, tanto en plantas emergidas como establecidas $(\mathrm{P}<0.001)$. En plantas emergidas (Cuadro 7) fueron mayor el diámetro de corona y la altura de planta $(\mathrm{P}<0.001)$ pero menor el número de tallos en pasto Rhodes que en Buffel. La siembra por cariópsides resultó en mayor diámetro de corona $(\mathrm{P}<0.01)$ y número de tallos $(\mathrm{P}<0.001)$ tanto en plantas emergidas como establecidas. Tapar el material de siembra y apisonarlo resultó en mayor diámetro de corona $(\mathrm{P}<0.001)$.

Cuadro 7. Diámetro de corona, altura de planta y número de tallos por planta de 2 gramíneas introducidas en Atotonilco El Grande, Hidalgo, México.

\begin{tabular}{|c|c|c|c|c|c|c|}
\hline \multirow[t]{2}{*}{ Tratamiento } & \multicolumn{3}{|c|}{ Plantas emergidas } & \multicolumn{3}{|c|}{ Plantas establecidas } \\
\hline & $\begin{array}{l}\text { Diám. corona } \\
(\mathrm{mm})\end{array}$ & $\begin{array}{l}\text { Altura planta } \\
(\mathrm{cm})\end{array}$ & $\begin{array}{l}\text { Tallos por } \\
\text { planta (no.) }\end{array}$ & $\begin{array}{l}\text { Diám. corona } \\
(\mathrm{mm})\end{array}$ & $\begin{array}{l}\text { Altura planta } \\
(\mathrm{cm})\end{array}$ & $\begin{array}{l}\text { Tallos por } \\
\text { planta (no.) }\end{array}$ \\
\hline \multicolumn{7}{|l|}{ Especie } \\
\hline Rhodes & $2.2 \mathrm{a}^{1}$ & $19.0 \mathrm{a}$ & $1.8 \mathrm{~b}$ & $18.0 \mathrm{~b}$ & $29.2 \mathrm{a}$ & $17.9 \mathrm{a}$ \\
\hline Buffel & $1.6 \mathrm{~b}$ & $5.7 \mathrm{~b}$ & $2.8 \mathrm{a}$ & $20.2 \mathrm{a}$ & $18.0 \mathrm{~b}$ & $10.2 \mathrm{~b}$ \\
\hline \multicolumn{7}{|l|}{ Material de siembra } \\
\hline Semilla cruda & $1.7 \mathrm{~b}$ & $11.3 \mathrm{a}$ & $1.9 \mathrm{~b}$ & $15.4 \mathrm{~b}$ & $25.5 \mathrm{a}$ & $10.6 \mathrm{~b}$ \\
\hline Cariópsides & $2.1 \mathrm{a}$ & $13.5 \mathrm{a}$ & $2.7 \mathrm{a}$ & $22.8 \mathrm{a}$ & $21.6 \mathrm{~b}$ & $17.6 \mathrm{a}$ \\
\hline \multicolumn{7}{|l|}{ Método de tapado } \\
\hline Testigo & $1.6 \mathrm{~b}$ & $11.7 \mathrm{a}$ & $2.2 \mathrm{a}$ & $15.5 b$ & $21.6 \mathrm{a}$ & $12.8 \mathrm{a}$ \\
\hline Ramas & $1.9 \mathrm{ab}$ & $11.1 \mathrm{a}$ & $2.0 \mathrm{a}$ & $17.8 \mathrm{~b}$ & $22.2 \mathrm{a}$ & $12.0 \mathrm{a}$ \\
\hline Rodillo & $2.0 \mathrm{a}$ & $13.4 \mathrm{a}$ & $2.6 \mathrm{a}$ & $21.7 \mathrm{a}$ & $26.1 \mathrm{a}$ & $15.6 \mathrm{a}$ \\
\hline Ramas + rodillo & $2.1 \mathrm{a}$ & $13.4 \mathrm{a}$ & $2.4 \mathrm{a}$ & $21.4 \mathrm{a}$ & $24.5 \mathrm{a}$ & $15.9 \mathrm{a}$ \\
\hline
\end{tabular}

${ }^{1}$ Valores en una misma columna y tratamiento seguidos de letras iguales no difieren en forma significativa $(\mathrm{P}<0.05)$. 
Cuadro 8. Diámetro de corona, altura de planta y número de tallos por planta de 2 gramíneas introducidas en Salinas Hidalgo, San Luis Potosí, México.

\begin{tabular}{|c|c|c|c|c|c|c|}
\hline \multirow{2}{*}{ Tratamiento } & \multicolumn{3}{|c|}{ Plantas emergidas } & \multicolumn{3}{|c|}{ Plantas establecidas } \\
\hline & $\begin{array}{l}\text { Diám. corona } \\
(\mathrm{mm})\end{array}$ & $\begin{array}{c}\text { Altura planta } \\
(\mathrm{cm})\end{array}$ & $\begin{array}{l}\text { Tallos por } \\
\text { planta (no.) }\end{array}$ & $\begin{array}{c}\text { Diám. corona } \\
(\mathrm{mm})\end{array}$ & $\begin{array}{l}\text { Altura planta } \\
(\mathrm{cm})\end{array}$ & $\begin{array}{l}\text { Tallos por } \\
\text { planta (no.) }\end{array}$ \\
\hline \multicolumn{7}{|l|}{ Especie } \\
\hline Rhodes & $6.3 \mathrm{a}^{1}$ & $49.0 \mathrm{a}$ & $9 \mathrm{a}$ & $15.6 \mathrm{~b}$ & $59.4 \mathrm{a}$ & $16 \mathrm{a}$ \\
\hline Buffel & $2.7 \mathrm{~b}$ & $6.3 b$ & $2 b$ & $25.7 \mathrm{a}$ & $17.3 b$ & $11 \mathrm{~b}$ \\
\hline \multicolumn{7}{|l|}{ Material de siembra } \\
\hline Semilla cruda & $3.8 \mathrm{~b}$ & $25.8 \mathrm{~b}$ & $5 b$ & $19.2 \mathrm{~b}$ & $38.2 \mathrm{a}$ & $13 \mathrm{a}$ \\
\hline Cariópsides & $5.2 \mathrm{a}$ & $29.6 \mathrm{a}$ & $6 a$ & $22.2 \mathrm{a}$ & $38.6 \mathrm{a}$ & $14 \mathrm{a}$ \\
\hline \multicolumn{7}{|l|}{ Método de tapado } \\
\hline Testigo & $4.1 \mathrm{a}$ & $24.5 \mathrm{~b}$ & $5 \mathrm{a}$ & $18.1 \mathrm{~b}$ & $36.8 \mathrm{a}$ & $12.5 \mathrm{~b}$ \\
\hline Ramas & $4.3 \mathrm{a}$ & $26.4 \mathrm{ab}$ & $6 a$ & $19.9 \mathrm{ab}$ & $38.7 \mathrm{a}$ & $13 \mathrm{ab}$ \\
\hline Rodillo & $4.8 \mathrm{a}$ & $29.3 \mathrm{ab}$ & $6 \mathrm{a}$ & $22.0 \mathrm{a}$ & $38.7 \mathrm{a}$ & $13 \mathrm{ab}$ \\
\hline Ramas + rodillo & $4.9 \mathrm{a}$ & $30.6 \mathrm{a}$ & $6 a$ & $22.7 \mathrm{a}$ & $39.3 \mathrm{a}$ & $15 \mathrm{a}$ \\
\hline
\end{tabular}

${ }^{1}$ Valores en una misma columna y tratamiento seguidos de letras iguales no difieren en forma significativa $(\mathrm{P}<0.05)$.

En Salinas Hidalgo, las plantas establecidas del pasto Buffel presentaron un incremento considerable del diámetro de corona en comparación con las plantas emergidas y superaron al Rhodes en esta característica (Cuadro 8) $(\mathrm{P}<0.01)$; no obstante este último presentó mayores valores de altura de planta y número de tallos $(\mathrm{P}<0.001)$. La siembra por cariópsides resultó en mayores valores en plantas emergidas para todas las características $(\mathrm{P}<0.001)$, mientras que en plantas establecidas la diferencia solo se presentó para diámetro de corona $(\mathrm{P}<0.01)$. Tanto en plantas emergidas como establecidas los mejores resultados se encontraron con material de siembra cubierto y apisonado.

\section{Discusión}

Quero-Carrillo et al. (2014) indican que en zonas áridas un establecimiento 'excelente' y un establecimiento 'aceptable' a 90 días post-siembra en praderas de temporal son de 30 y 8 plantas $/ \mathrm{m}^{2}$, respectivamente. Posteriormente y debido a competencia, a 36 meses estos niveles son de 6 y 2 plantas $/ \mathrm{m}^{2}$, respectivamente. En Atotonilco El Grande y en Salinas Hidalgo no se observaron diferencias entre sitios $(\mathrm{P}>0.05)$. La emergencia y el establecimiento fueron muy bajos, apenas 'aceptables', tanto para las gramíneas nativas como las introducidas. En el primer sitio la relación entre el número de plantas establecidas $/ \mathrm{m}^{2}$ y el número de plantas emergidas $/ \mathrm{m}^{2}$ fue 2:10 para las especies nativas y 2:11 para las introducidas. En el segundo sitio estos valores fueron 1:4 y 2:9, respectivamente. Estos resultados contrastan considerablemente con el número de plantas esperadas (>1,000 plantas $/ \mathrm{m}^{2}$, según los datos en el Cuadro 1). Esto se debe probablemente a las condiciones de tempe- ratura y humedad que resultaron en la deshidratación de plántulas, debido a la elongación del entrenudo subcoleoptilar, el cual, al contener el meristemo apical generador de raíces adventicias que sustentan la supervivencia de la planta, provocó que éstas se formaran cerca de la superficie del suelo donde las condiciones ambientales fueron desfavorables para el desarrollo de plántulas (Moreno-Gómez et al. 2012).

Respecto al factor precipitación en zonas áridas, Cox y Jordan (1983) en Arizona registraron solo 0.6\% de establecimiento para Eragrostis lehmanniana y consideraron que este bajo valor fue debido a la escasa y mala distribución de la precipitación. Así mismo Ward et al. (2006) encontraron en estudios de invernadero que para la germinación de semillas de Buffel se requiere, como mínimo, la aplicación de $2 \mathrm{~mm}$ de agua durante 4 días de riego por goteo. En la presente investigación, la cantidad de lluvia no pareció ser un factor limitante para el establecimiento, ya que 3 días después de la siembra se registraron $30 \mathrm{~mm}$ de precipitación en Atotonilco El Grande (Cuadro 2), mientras que en Salinas Hidalgo es un factor a considerar. La intensidad y distribución errática de la lluvia, características de zonas áridas, afecta el establecimiento de las plantas emergidas: La precipitación en verano en ambos sitios influyó para registrar menor cantidad de plantas establecidas por la pobre retención de humedad en suelos de bajo contenido de materia orgánica, lo que afecta la emergencia y establecimiento de plántulas (Cibrián-Tovar et al. 2013; QueroCarrillo et al. 2014).

Por otro lado, Larsen y Bibby (2004) y Ward et al. (2006) consideran que la temperatura adecuada es fundamental para el establecimiento de pasturas perennes en zonas áridas. La fluctuación de la temperatura afectó la 
germinación de los cultivares en estudio, debido a que son plantas de metabolismo $\mathrm{C}_{4} \mathrm{y}$ la temperatura mínima para germinación debe estar alrededor de $10{ }^{\circ} \mathrm{C}$ (Ward et al. 2006). En la región la temperatura en la superficie del suelo varía entre 5 y $30{ }^{\circ} \mathrm{C}$, pero puede alcanzar valores entre 5 y $15{ }^{\circ} \mathrm{C}$ por efecto de siembra y tapado de las semillas (Evans y Young 1987). En este estudio el promedio de temperaturas mínimas fue de $13.2{ }^{\circ} \mathrm{C}$ en Atotonilco El Grande y $14{ }^{\circ} \mathrm{C}$ en Salinas Hidalgo, 5 días después de la siembra, aunque con algunas fluctuaciones. Hernández-Guzmán et al. (2015) en condiciones de laboratorio $\left(22{ }^{\circ} \mathrm{C}\right)$ observaron germinaciones altas de cariópsides clasificadas por tamaño de las mismas especies de este estudio, así: en Banderita y Navajita $>80 \%$ y en Rhodes y Buffel $>55 \%$ y $28 \%$, respectivamente. Estas notorias diferencias en la emergencia entre campo y condiciones de laboratorio corroboran la importancia de temperaturas más elevadas, las cuales, sin embargo, en los sitios del estudio ocurren en abril-mayo cuando no hay disponibilidad de humedad adecuada. La sequía intraestival también influye en la reducción del número de plántulas emergidas y establecidas posterior a la siembra (Quero et al. 2007).

Las heladas tempranas, por otra parte, también afectan el establecimiento, especialmente cuando existe bajo desarrollo de plántulas y la siembra se realiza a finales de julio. Cox et al. (1986) tuvieron poco éxito en el establecimiento de Eragrostis lehmanniana y E. curvula debido a que la mayoría de plántulas no sobrevivieron a temperaturas bajas $\left(<3{ }^{\circ} \mathrm{C}\right)$ por su baja capacidad para almacenar reservas de carbohidratos en raíz y corona. En el presente estudio los mayores diámetros de corona, altura de planta y número de tallos ocurrieron en Salinas Hidalgo en comparación con los mismos valores obtenidos en Atotonilco El Grande, posiblemente como resultado de la mayor radiación solar en el primer sitio durante la temporada de crecimiento activo de la plántula. En Atotonilco El Grande entre julio y octubre de 2010 se registraron $1,684 \mathrm{Watt} / \mathrm{m}^{2}$ y en $2011,1,892 \mathrm{Watt} / \mathrm{m}^{2}$; mientras que en Salinas Hidalgo se registraron 2,157 $\mathrm{Watt} / \mathrm{m}^{2}$ en 2010 y $2,150 \mathrm{Watt} / \mathrm{m}^{2}$ en 2011.

Según Cox y Jordan (1983), Jordan y Maynard (1970) y Cox et al. (1982), para mejorar el establecimiento de pasturas en zonas de escasa precipitación, es importante hacer una adecuada preparación de la cama de siembra con el fin de favorecer la germinación de las semillas y el establecimiento de las plántulas. En este estudio, esto se logró en los tratamientos que incluían el tapado y apisonado de las semillas crudas y cariópsides. La baja emergencia en las siembras con cariópsides de especies nativas e introducidas puede ser el resultado de la depredación de las semillas botánicas por hormigas, aves y pequeños mamíferos (Whitford 2002).

\section{Conclusiones}

- En las especies nativas Navajita (Bouteloua gracilis) y Banderita (B. curtipendula) el mayor número de plantas emergidas y establecidas se alcanzó cuando la siembra se realizó con semilla cruda. En las especies introducidas Buffel (Cenchrus ciliaris) y Rhodes (Chloris gayana) los resultados fueron similares, independiente del material de siembra utilizado (semilla cruda o cariópsides).

- Las prácticas de tapado y apisonado de semillas después de la siembra resultaron en mayor cantidad de plantas emergidas así como mayor diámetro de corona, lo que es importante para acumular sustancias de reserva que ayuden a sobrevivir las épocas de invierno y a rebrotar en las primeras lluvias.

- La cantidad de precipitación aparentemente no fue factor decisivo para establecer las especies estudiadas, mientras que la distribución de la precipitación y la fluctuación de temperatura en ambos sitios aparentemente fue determinante para la menor emergencia de plántulas de las especies sembradas.

\section{Referencias}

Cibrián-Tovar J; Quero-Carrillo AR; Muñiz-Merino M; Hernández-Guzmán FJ; Cibrián-Jaramillo AM; ManzanoCamarillo M. 2013. Nanotecnología agrícola: Caso 1. Liberadores de ferohormonas, Caso 2. Establecimiento de gramíneas en condiciones semiáridas. Agroproductividad 6:3-7. http://goo.gl/JFdTzT

Cox JR; Morton HL; Johnsen Jr TN; Jordan GL; Martin SC; Fierro LC. 1982. Vegetation restoration in the Chihuahuan and Sonoran deserts of North America. Agricultural Reviews and Manuals, Western Series No. 28. USDA Agricultural Research Service, Tucson, AZ, USA. http://goo. gl/oggigi

Cox JR; Jordan GL. 1983. Density and production of seeded range grasses in southeastern Arizona (1970-1982). Journal of Range Management 36:649-652. DOI: $\underline{10.2307 / 389}$ $\underline{8361}$

Cox JR; Martin-R MH; Ibarra-F FA; Morton HL. 1986. Establishment of range grasses on various seedbeds at creosotebush (Larrea tridentata) sites in Arizona, U.S.A., and Chihuahua, Mexico. Journal of Range Management 39:540-546. DOI: 10.2307/3898767

Esqueda Coronado MH; Carrillo Romo RL; Sosa Cerecedo M; Melgoza Castillo A; Royo Márquez MH; Jiménez Castro J. 2002. Emergencia y sobrevivencia de gramíneas inoculadas con biofertilizantes en condiciones de invernadero. 
Técnica Pecuaria en México 42:459-475. http://goo.gl/VS AawR

Evans RA; Young JA. 1987. Seedbed microenvironment, seedling recruitment, and plant establishment on rangelands. En: Frasier GW; Evans RA, eds. Proceedings of the Symposium "Seed and Seedbed Ecology of Rangeland Plants", 21-23 April 1987, Tucson, AZ. Aridland Watershed Management Research Unit, ARS-USDA, Tucson, AZ, USA. p. 52. http://goo.gl/QYZ0Vs

FAO-UNESCO-ISRIC (Food and Agriculture Organization United Nations Educational, Scientific and Cultural Organization - International Soil Reference and Information Centre). 1997. Soil map of the world. Revised legend with corrections and updates. World Resources Report 60. Roma, Italia. (Reimpresión actualizada como Technical Paper 20, ISRIC, Wageningen, Países Bajos). http://goo.gl/8ZN6aq

Hernández-Guzmán FJ; Quero-Carrillo AR; Pérez-Rodríguez P; Velázquez-Martínez M; García-de los Santos G. 2015. Germinación y emergencia de propágulos de pasto, en respuesta a pruebas de vigor. Revista Mexicana de Ciencias Agrícolas 6:1519-1532. http://goo.gl/ocGexO

INEGI (Instituto Nacional de Estadística, Geografía e Informática). 1984. Cartas cartográficas del Estado de Hidalgo, México.

INEGI (Instituto Nacional de Estadística, Geografía e Informática). 2012. Cuaderno Municipal de Soledad de Graciano Sánchez, Estado de San Luis Potosí, México.

ISTA (International Seed Testing Association). 1996. International rules for seed testing. Seed Science and Technology 24, Supplement.

Jordan GL; Maynard ML. 1970. The San Simon watershed: Revegetation. Progressive Agriculture in Arizona 22(6): 4-7. http://goo.gl/5Tn9aB

Kottek M; Grieser J; Beck C; Rudolf B; Rubel F. 2006. World map of the Köppen-Geiger climate classification updated. Meteorologische Zeitschrift 15:259-263. DOI: $\underline{10.1127 / 09}$ 41-2948/2006/0130

Larsen SU; Bibby BM. 2004. Use of germination curves to describe variation in germination characteristics in three turfgrass species. Crop Science 44:891-899. https://goo.gl/ E6Hkpf

Moreno-Gómez B; García-Moya E; Rascón-Cruz Q; AguadoSantacruz GA. 2012. Crecimiento y establecimiento de plántulas de Bouteloua gracilis (Kunth) Lag. ex Griffiths y Eragrostis curvula var. conferta Stapf bajo un régimen simulado de lluvia. Revista Fitotecnia Mexicana 35:299-308. http://goo.gl/SN6AkB

PMAR (Plan Maestro de la Alianza Regional). 2012. Plan Maestro de la Alianza Regional para la Conservación de los Pastizales del Desierto Chihuahuense. En: GuzmánAranda JC; Hoth J; Berlanga H, eds. Comisión para la Cooperación Ambiental. Montreal, QC, Canadá.

Probert RJ; Hay FR. 2000. Keeping seeds alive. En: Black M; Bewley JD, eds. Seed technology and its biological basis. Sheffield Academic Press, Sheffield, UK. p. 390-393.

Quero Carrillo AR; Enríquez Quiroz JF; Miranda Jiménez L. 2007. Evaluación de especies forrajeras en América tropical, avances o status quo. Interciencia 32:566-571. http://ref.scielo.org/js8x3f

Quero-Carrillo AR; Miranda-Jiménez L; Hernández-Guzmán FJ; Rubio-Aguirre F. 2014. Mejora del establecimiento de praderas. Folleto Técnico. Colegio de Postgraduados. Montecillo, Texcoco, Estado de México, México.

SAS (Statistical Analysis System). 2009. SAS/STAT User guide version 9.1.3. SAS Institute Inc. Cary, NC, USA.

Tian X; Knapp AD; Moore KJ; Brummer EC; Bailey TB. 2002. Cupule removal and caryopsis scarification improves germination of eastern gamagrass seed. Crop Science 42:185-189. DOI: $10.2135 /$ cropsci2002.0185

Townsend PA; Ortega HMA; Bartley J; Sánchez CV; Soberón J; Buddemeier RH; Stockwell DRB. 2002. Future projections for Mexican faunas under global climate change scenarios. Nature 416:626-629. DOI: 10.1038/416626a

Ward JP; Smith SE; McClaran MP. 2006. Water requirements for emergence of buffel grass (Pennisetum ciliare). Weed Science 54:720-725. DOI: $10.1614 / \mathrm{ws}-05-163 \mathrm{r} 1.1$

Whitford W. 2002. Ecology of desert systems. Academic Press, Elsevier Science, San Diego, CA, USA.

(Received for publication 17 June 2015; accepted 31 August 2015)

(C) 2016

\section{(c) (i) (2) (2)}

Tropical Grasslands-Forrajes Tropicales is an open-access journal published by Centro Internacional de Agricultura Tropical (CIAT). This work is licensed under a Creative Commons Attribution-NonCommercial-ShareAlike 3.0 Unported License. To view a copy of this license, visit http://creativecommons.org/licenses/by-nc-sa/3.0/ 